\title{
THE PRESENCE OF ANTI-PHOSPHATIDYLETHANOLAMINE ANTIBODIES IN ACUTE MYOCARDIAL INFARCTION
}

\author{
1,2 Abdolreza Sotoodeh Jahromi, \\ ${ }^{1}$ Mohammad Shojaei, ${ }^{3}$ Mohammad Reza Farjam and ${ }^{4}$ Abdolhossien Madani \\ ${ }^{1}$ Atherosclerosis Research Center, \\ ${ }^{2}$ Department of Immunology, \\ ${ }^{3}$ Research Center for Social Determinants of Health, \\ Faculty of Medicine, Jahrom University of Medical Science, Jahrom, Iran \\ ${ }^{4}$ Research Center for Social Determinants in Health Promotion, \\ Faculty of Public Health, Hormozgan University of Medical Science, Bandarabbas, Iran
}

Received 2013-09-29, Revised 2013-10-19; Accepted 2013-10-21

\begin{abstract}
Acute Myocardial Infarction (AMI) is a clinical manifestation of coronary atherothrombosis and is the important causes of death. Many factors play a role in AMI. Anti-Phospholipid (aPL) antibodies may act in the induction of immunological response leading to the development of AMI. AntiPhosphatidylethanolamine (aPEA) antibody has been detected in various autoimmune diseases and anti-phospholipid antibody syndrome. The study of aPEA antibody in AMI might shed light on etiologic mechanisms in the pathogenesis of coronary atherothrombosis and AMI. This study was aimed to evaluate whether prevalence of aPEA antibodies, in patients with AMI and to analyze their relationship with traditional cardiovascular risk factors. The prevalence of aPEA IgG and IgM in a well characterized group of patients with AMI as a case group and in age and sex matched healthy subjects as a control group. Sera from two groups were tested to evaluate the presence of aPEA IgG and $\operatorname{IgM}$ isotypes by ELISA method. The frequencies of positive test for aPEA IgG were 12.22 and $2.22 \%$ among patients and controls respectively with significant difference $(p=0.007)$. The aPEA IgM frequencies were 3.33 and $0.00 \%$ in patients and the controls, with significant difference $(\mathrm{p}=0.005)$. According to the results of this study, aPEA antibodies have a role in AMI, independent risk factors for AMI, which may represent a link between autoimmunity and coronary atherothrombosis. Further studies with larger sample size of patients and healthy people are needed to explore the role of aPEA antibodies in coronary atherothrombosis.
\end{abstract}

Keywords: Anti-Phosphatidylethanolamine (aPEA) Antibodies, Acute Myocardial Infarction (AMI), AntiPhospholipid (aPL) Antibodies, Cardiovascular Ischemia, Coronary Atherothrombosis

\section{INTRODUCTION}

Acute Myocardial Infarction (AMI) is a clinical manifestation of coronary atherothrombosis and among Cardiovascular Diseases (CVD) is the important causes of morbidity and mortality (Upaganlawar et al., 2011).

Arterial thrombosis (myocardial infarction, cerobrovascular accident, angina) is the collective result of environmental factors and personal Corresponding Author: Mohammad Reza Farjam, Research Center for Social Determinants of Health, Faculty of Medicine, Jahrom University of Medical Science, Jahrom, Iran 
predispositions. Factors such as low serum adiponectin (Shojaie et al., 2009a) and low annexin V levels (Shojaie et al., 2009b) and infectious diseases such as Mycoplasma pneumonia (Pourahmad et al., 2009) are a part of involving factors in AMI. It has been demonstrated that the immunopathological such as anti-beta2 glycoprotein-I antibody (Shojaie et al., 2011), Anti-Phosphatidylserine antibodies (Jahromi et al., 2013) and Inflammatory processes (Jahromi et al., 2010a) have important roles in the initiation and development of AMI.

It seems that several subspecificities of antiphospholipid antibodies may occur in the patients with MI and some evidence suggest that these antibodies may contribute to the development of MI (Vaarala, 1998). However their pathogenic mechanisms in AMI are still matter of research.

Besides the Classical Lupus Anticoagulant (LAC) and Anti-Cardiolipin Antibodies (ACA), other antiPhospholipid Antibodies (aPL) were shown to target anionic phospholipids and other plasma proteins, including phosphatidylethanolamine (PEA), protein C, protein $\mathrm{S}$, B2-Glycoprotein $\mathrm{I}$ and annexin $\mathrm{V}$ (Tincani et al., 2010).

Venous thrombosis is the most common vascular event; however, one third patients with Antiphospholipid Syndrome (APS) presents arterial thrombosis (myocardial infarction, cerobrovascular accident, angina) during the progression of the disease (Matsuura and Lopez, 2008).

Elevated serum levels of antiphospholipid antibodies along with thromboembolic complications involving arterial blood vessels (Shojaie et al., 2011; Jafarzadeh et al., 2011) or with pregnancy morbidity (miscarriages and fetal loss) represent the major features of APS (Jahromi et al., 2010b).

It is notable that genetic factors as well as other traditional risk factors such as smoking, hypercholesterolemia, diabetes mellitus and hypertension may contribute to IHD development and these parameters differ among various population. Even though there are a few studies on the association of some autoantibodies with AMI, more epidemiological data are needed to confirm their significance as independent risk factors in CVD. Furthermore, the data on the correlation of autoantibodies with traditional risk factors of AMI is scarce.
Henceforth, present case control study was undertaken to evaluate the serum levels of antiPhosphatidylethanolamine (aPEA) antibodies (IgG and $\operatorname{IgM}$ ) in Iranian patients with AMI and also to illuminate their association with traditional risk factors of this disease.

\section{MATERIALS AND METHODS}

\subsection{Subjects}

A total of 90 consecutive patients (aged 41-67 years) with AMI including 67 men and 23 women who were admitted to Peymanieh Hospital of Jahrom (a southwest city of Iran), were enrolled to this casecontrol study.

AMI was diagnosed by the presence of two of the following criteria: (i) prolonged chest pain compatible with AMI, (ii) typical ECG changes, (iii) rising of cardiac enzymes such as creatine kinase and lactate dehydrogenase (Antman et al., 2008).

Exclusion criteria were valvular heart disease, surgery, trauma during the prior month, cardiomyopathy, liver disease, renal failure, arthritis, malignant diseases, other inflammatory diseases (such as SLE and RA) and oral anticoagulant therapy. In patients with AMI, the serum concentration of AntiPC antibodies was measured during 3-5 days after admission.

An age, sex and other CAD risk factors such as Hypertension (HTN), Diabetes Mellitus (DM) and Hyper Lipidemia (HLP) matched group $(n=90)$ with similar geographic and socioeconomic backgrounds without any ischemic heart disease were used as a control group. All control subjects were basically healthy, with no acute or chronic illnesses and did not use any drugs. The healthy control group was recruited from blood donors attending Jahrom Blood Transfusion Center.

The study protocol was approved by research ethics committee of Jahrom University of Medical Sciences and informed consents were obtained from all participants before enrollment. The patient or his/her legal representative provided written informed consent.

Historical, demographic and clinical data were obtained through a review of medical records and 
interviews with patients and their families. The risk factors for myocardial infarction were as following: (a) age, sex; (b) history of hypertension (diagnosis confirmed when the systolic or diastolic pressures were $>160$ or $95 \mathrm{mmHg}$, respectively, or when the patient was using antihypertensive medication) 12; (c) smoking, according to the criteria of the British Council for Medical Research; (d) history of heart disease (atrial fibrillation or coronary heart disease, defined as previous myocardial infarction, angina, or revascularization procedure); (e) history of diabetes mellitus, according to the medical history or the use of insulin or an oral anti-diabetes drug; (f) hypercholesterolemia, based on total cholesterol $>200 \mathrm{mg} / \mathrm{dL}$, LDL-cholesterol $>130$ $\mathrm{mg} / \mathrm{dL}$, or total cholesterol/HDL-cholesterol ratio $>5$ (Donahue et al., 1988).

Blood samples (5 cc) were obtained by venipuncture from the patients immediately after admission before starting any IV medications by skilled personnel. Blood samples were centrifuged and frozen within, at most, $2 \mathrm{~h}$ after collection and stored at $-20^{\circ} \mathrm{C}$ until laboratory testing.

Enzyme-immunoassay kits (BINDAZYME Human Anti-phosphatidylethanolamine IgG/IgM Combi EIA Kit, MK077, manufactured by: The Binding Site Ltd., Birmingham, U.K.) were used for the detection of IgG or IgM aPEA by indirect solid phase enzyme immunoassay (ELISA) as suggested by the ELISA kit manufacturer.

Statistical analyses were performed by SPSS (version 17; SPSS, Inc., Chicago, IL). Data were expressed as mean \pm SD. Continuous variables with little-to-mild skewness were summarized as mean \pm SD and compared using Student's t-test.

\section{RESULTS}

The demographic and clinical characteristics of the study groups, as well as laboratory variables are shown in Table 1.

In the patient group 17 cases $(18.89 \%)$ had Non-St Elevation MI (NSTEMI) and $73(81.11 \%)$ had ST Elevation MI (STEMI).

There was no significant difference between the two groups regarding the following variables: age, sex, HTN, DM, LDL, HDL, total cholesterol and TG.

The association between positive aPEA IgG and IgM tests and selected cardiovascular risk factors were examined in this study.

There was a significant association between positive aPEA IgG and IgM tests with hypertension. But there was not found any significant association between positive aPEA IgG and IgM tests with Type-I DM, TypeII DM, age, sex, LDL, HDL, TG, total cholesterol and adjusted smoking.

There was not found significant difference between positive aPEA IgG test in patients with STEMI and those with NSTEMI $(74.40 \%$ Vs $24.80 \%)$, $(p=0.154)$ and also there was not found significant difference between positive aPEA IgM test in patients with STEMI and those with NSTEMI (67.10\% Vs 23.70\%), $(\mathrm{p}=0.434)$.

Table 1. Demographic, clinical characteristics and laboratory finding for the case and the control groups

\begin{tabular}{llll}
\hline Variable & Patients groups & Control group & P-Value \\
\hline Age (year) & $62.76 \pm 10.53$ & $61.76 \pm 9.52$ & 0.657 \\
Male (\%) number & $68(75.60 \%)$ & $67(74.40 \%)$ & 0.863 \\
High blood pressure (\%) numbers & $40(44.40 \%)$ & $32(35.60 \%)$ & 0.287 \\
Smoker (\%) number & $39(43.33 \%)$ & $28(31.10 \%)$ & 0.078 \\
IDD*(\%) number & $24(26.70 \%)$ & $28(31.10 \%)$ & 0.612 \\
Total cholesterol* Mgdl & $175.13 \pm 41.30$ & $167.80 \pm 42.20$ & 0.114 \\
HDL-C* Mg/dL & $45.20 \pm 9.110$ & $43.83 \pm 10.29$ & 0.338 \\
LDL-C* Mg/dL & $119.05 \pm 35.32$ & $99.22 \pm 29.64$ & 0.095 \\
TG*Mg/dL & $127.54 \pm 73.18$ & $113.84+42.52$ & 0.106 \\
Positive Apea IgG (\%) number & $11(12.22 \%) 0$ & $(2.22 \%) 2.00$ & 0.007 \\
Positive Apea IgG $(\%)$ number & $(3.33 \%) 300$ & $(0.0 \%) 0.000$ & 0.005 \\
\hline
\end{tabular}

*IDDM = Insuline Dependent Diabetes Mellitus, NIDDM = Non-Insuline Dependent Diabetes Mellitus, HDL-C = High Density Lipoprotein-Cholesterol, LDL-C = Low Density Lipoprotein-Cholesterol, FBS = Fasting Blood Sugar, aPEA = Anti-Phatidylethanol amine 


\section{DISCUSSION}

The results of the present study showed that the prevalence of positive aPEA IgG and also IgM in patients with AMI were significantly higher than that detected in healthy control group. These associations were independent of traditional risk factors. Other aPLs antibodies have been reported to have association with CAD and AMI (Shojaie et al., 2011; Jafarzadeh et al., 2011; Greco et al., 2010). The result of present study and other researches (Shojaie et al., 2011; Jafarzadeh et al., 2011; Urbanus et al., 2009; Jahromi et al., 2013; Sanmarco et al., 2007) are consistent with current concepts on the immune pathogenesis of atherosclerosis. However, the precise mechanisms of involvement of aPEA antibodies in the pathogenesis of AMI remain to be determined.

The presence of increased titers of aPEA antibodies in our patients were independent factors for AMI and were not associated with any of the conventional cardiovascular risk factors (smoking, hypertension, mellitus and hypercholesterolemia). These results are in accordance with the results of previous studies (Shojaie et al., 2011; Jahromi et al., 2010c; 2013).

In this study there was a significant association between aPEA IgG with HTN in the case and the control groups. These results suggest that aPEA IgG are independent of these variables.

As there were not significant differences between positive aPEA antibodies test in patients with STEMI and those with NSTEMI, it can be concluded that aPEA IgG and IgM do not participate in kind of AMI (STEMI Vs NSTEMI). But, unfortunately, there were not found any data indicating association of aPEA $\operatorname{IgG}$ and $\operatorname{IgM}$ with these parameters to compare the results.

\section{CONCLUSION}

These results show an association between AMI and high prevalence of positive tests for aPEA IgG and IgM. The results of this study in parallel with the data from other studies about oyher anti-phospholipid antibodies (Shojaie et al., 2011; Jafarzadeh et al., 2011; Urbanus et al., 2009; Jahromi et al., 2013; Sanmarco et al., 2007) support the potential important role of aPL antibodies in AMI.

According to the result of this study, it seems that aPEA antibodies are associated with AMI and may involve in the development of AMI.
Clinical trials for the prevention of myocardial infarction and atherosclerosis in patients with antiphospholipid antibodies are recommended. Further studies with larger sample size of patients and healthy people are needed to explore the exact role of aPEA IgG and IgM in AMI. To achieve this, these kinds of studies should be planned as international multicenter researches.

\section{ACKNOWLEDGEMENT}

This study was completely financed by Jahrom University of Medical Sciences. The authors are grateful to the patients and the control individuals who accepted to enter this study.

\section{REFERENCES}

Antman, E.M., M. Hand, P.W. Armstrong, E.R. Bates and L.A. Green et al., 2008. STEMI focused update: 2007 Focused Update of the ACC/AHA 2004 Guidelines for the Management of Patients With ST-Elevation Myocardial Infarction: A report of the American College of Cardiology/American Heart Association Task Force on Practice Guidelines: Developed in collaboration With the Canadian Cardiovascular Society endorsed by the American Academy of Family Physicians: 2007 Writing Group to Review New Evidence and Update the ACC/AHA 2004 Guidelines for the Management of Patients With STElevation Myocardial Infarction, Writing on Behalf of the 2004 Writing Committee. Circulation, 15: 296$329 . \quad$ DOI: 10.1161/ CIRCULATIONAHA.107.188209

Donahue, R.P., R.D. Abbott, D.M. Reed and K. Yano, 1988. Physical activity and coronary heart disease in middle-aged and elderly men: The honolulu heart program. Am. J. Publ. Health, 78: 683-685. DOI: 10.2105/AJPH.78.6.683

Greco, T.P., A.M. Conti-Kelly, J.R. Anthony, T. Greco and R. Doyle et al., 2010. Oxidized-LDL $/ \beta_{2}-$ glycoprotein i complexes are associated with disease severity and increased risk for adverse outcomes in patients with acute coronary syndromes. Am. J. Clin. Pathol., 133: 737-743. DOI: 10.1309/AJCP88WVRDRDFBAS

Jafarzadeh, A., M. Poorgholami, M. Nemati and M. Rezayati, 2011. High serum levels of rheumatoid factor and anti-phosphatidylserine antibody in patients with ischemic heart disease. Iran. J. Immunol., 8: 34-44. PMID: 21427494 
Jahromi, A.S., M. Shojaei, M.R. Farjam and A. Madani, 2013. Anti-phosphatidylserine antibodies in acute myocardial infarction. Am. J. Immunol., 9: 96-100. DOI: 10.3844/ajisp.2013.96.100

Jahromi, A.S., M. Shojaie and A. Madani, 2010a. Cardiotrophin-1 in patients with acute myocardial infarction. Am. J. Applied Sci., 7: 1190-1194. DOI : 10.3844/ajassp.2010.1190.1194

Jahromi, A.S., M. Shojaie, S. Dana and A. Madani, 2010c. Anti-cardiolipin antibody in acute myocardial infarction. Am. J. Immunol., 6: 11-14. DOI: 10.3844/ajisp.2010.11.14

Jahromi, A.S., M.R. Farjam, F. Mogharrab, A. Daryanavard and A. Madani, 2010b. Anti $\beta 2-$ glycoprotein i antibodies in women with recurrent spontaneous abortion. Am. J. Biochem. Biotechnol., 6: 264-267. DOI: 10.3844/ajbbsp.2010.264.267

Matsuura, E. and L.R. Lopez, 2008. Autoimmunemediated atherothrombosis. Lupus, 17: 878-887. DOI: 10.1177/0961203308093553

Pourahmad, M., A.S. Jahromy and M. Shojaei, 2009. Association of Mycoplasma pneumoniae infection with myocardial infarction. Am. J. Immunol., 5: 8488. DOI: 10.3844 /ajisp.2009.84.88

Sanmarco, M., S. Gayet, M.C. Alessi, M. Audrain and E. de Maistre et al., 2007. antiphosphatidylethanolamine antibodies are associated with an increased odds ratio for thrombosis. A multicenter study with the participation of the European Forum on antiphospholipid antibodies. Thromb. Haemost, 97: 949-954. PMID: 17549297
Shojaie, M., A. Sotoodah and G. Shafaie, 2009a. Is adiponectin associated with acute myocardial infarction in Iranian non obese patients? Lipids Health Dis., 8: 17-17. DOI: 10.1186/1476-511X-817

Shojaie, M., A. Sotoodah, S. Roozmeh, E. Kholoosi and S. Dana, 2009b. Annexin V and anti-annexin V antibodies: Two interesting aspects in acute myocardial infarction. Thromb. J., 21: 7-13. DOI: 10.1186/1477-9560-7-13

Shojaie, M., A.S. Jahromi, H. Ebadat, S.H. Moosavy and B. Seddigh et al., 2011. Anti- $\beta_{2}$ glycoprotein-i antibody in acute myocardial infarction. Am. J. Applied Sci., 8: 758-761. DOI: 10.3844/ajassp.2011.758.761

Tincani, A., C. Casu, S. Cartella, T. Ziglioli and R. Cattaneo, 2010. Antiphospholipid antibody: Laboratory, pathogenesis and clinical manifestations. Reumatismo, 62: 65-75. PMID: 20390120

Upaganlawar, A., H. Gandhi and R. Balaraman, 2011. Isoproterenol induced myocardial infarction: Protective role of natural products. Am. J. Pharmacol. Toxicol., 6: 1-17. DOI: 10.3923/jpt.2011.1.17

Urbanus, R.T., B. Siegerink, M. Roest, F.R. Rosendaal and de P.G. Groot, 2009. Antiphospholipid antibodies and risk of myocardial infarction and ischaemic stroke in young women in the RATIO study: A case-control study. Lancet Neurol., 8: 9981005. DOI: $10.1016 / \mathrm{S} 1474-4422(09) 70239-\mathrm{X}$

Vaarala, O., 1998. Antiphospholipid antibodies and myocardial infarction. Lupus, 7: S132-132. DOI: 10.1177/096120339800700229 